

\title{
TENDENCIAS DE INVESTIGACIÓN EN ARQUITECTURA ENTRE 2016-2020 EN LA BASE DE DATOS SCOPUS Y SU RELACIÓN CON LA CREACIÓN DE GRUPOS DE INVESTIGACIÓN
}

\author{
ARCHITECTURE RESEARCH TRENDS BETWEEN 2016-2020 IN THE \\ SCOPUS DATABASE, AND THEIR RELATIONSHIP WITH THE CREATION \\ OF RESEARCH GROUPS
}

\author{
CLAUDIA COSTA-DE LOS REYES \\ Magíster en Construcciones \\ Docente de la Escuela de Arquitectura \\ Universidad Internacional del Ecuador \\ Loja, Ecuador \\ https://orcid.org/0000-0001-6389-3094 \\ clcostade@uide.edu.ec \\ MARÍA ISABEL VIVANCO-VILLAVICENCIO \\ Magíster en Hábitat Sustentable y Eficiencia Energética \\ Docente de la Escuela de Arquitectura \\ Universidad Internacional del Ecuador \\ Loja, Ecuador \\ https://orcid.org/0000-0002-3773-6981 \\ mavivancovi@uide.edu.ec
}

\author{
SILVIA VIÑÁN-LUDEÑA \\ Magíster en Arquitectura \\ Docente de la Escuela de Arquitectura \\ Universidad Internacional del Ecuador \\ Loja, Ecuador \\ https://orcid.org/0000-0002-5600-6159 \\ sivinanlu@uide.edu.ec \\ FERNANDO MONCAYO-SERRANO \\ Magíster en Construcciones \\ Docente de la Escuela de Arquitectura \\ Universidad Internacional del Ecuador \\ Loja, Ecuador \\ https://orcid.org/0000-0002-8053-4541 \\ femoncayose@uide.edu.ec
}

(n) 


\section{INTRODUCCIÓN}

El Departamento de Investigación y Postgrados de la Universidad Internacional del Ecuador (UIDE) plantea la formación de grupos de investigación constituidos inicialmente por docentes de la institución; los objetivos de estos grupos son organizar los procesos de investigación para mejorar la participación de los investigadores y subir los índices académicos relacionados con la producción científica.

Ante la necesidad de formar un grupo de investigación en Arquitectura, se ha propuesto inicialmente aplicar técnicas bibliométricas con el fin de encontrar focos de investigación que servirán para articular los intereses de los diferentes perfiles docentes con un tema macro que direccione el trabajo del equipo.

En lo que se refiere a los grupos de investigación, las exigencias actuales del desarrollo de conocimiento científico por su complejidad se han extendido más allá de la comprensión individual especializada, por lo que se requiere complementariedades y sinergias desde la visión de diferentes investigadores (Smith, Vacca, Krenz y Mc Carty, 2021).

En ese sentido, dentro del contexto de la Escuela de Arquitectura de la UIDE, a pesar de que los integrantes principales del futuro grupo de investigación tengan en su mayoría el mismo título profesional, la especialidad difiere, siendo los más frecuentes los relacionados con la arquitectura, la construcción y proyectos arquitectónicos, por lo que es necesario establecer un proceso objetivo de selección de los temas principales de investigación con el propósito de que los participantes aporten desde sus campos de especialidad. Esto permitirá desarrollar valores y objetivos del grupo de investigación basados en la innovación, la cooperación interna, la pertinencia y la calidad de la producción académica, tal como la bibliografía revisada informa (Agnete, Aina, Svein y Ingvild, 2016).

Por otro lado, es preciso aclarar que dentro de la organización interna de la Escuela de Arquitectura ya existe un grupo de investigación que trata los temas urbanos y territoriales, por tanto, esta investigación se centra en los temas relacionados con arquitectura y edificación, sin embargo, los integrantes del grupo de investigación existente también fueron considerados para este estudio.

El dinamismo de la investigación dentro de las universidades ha puesto en evidencia la necesidad de creación de grupos de investigación y planteamiento de líneas de investigación que permitan enfocar el trabajo en temas relevantes para la sociedad. En este contexto, analizar las tendencias de investigación dentro de una disciplina o área de conocimiento, es muy importante y ha dado lugar a diversas revisiones sistemáticas de literatura especializada y estudios bibliométricos que, a través de indicadores, permiten evaluar la ciencia y la productividad científica (Gallardo, 2016), además de orientar a la comunidad científica y académica respecto a los temas relevantes que deben ser investigados.

Asimismo, los medios de comunicación científica se van consolidando desde las revistas internacionales y de acceso abierto. En ese sentido, a la vez que crece exponencialmente dicha producción, también se van generando interfaces de ambiente web con el propósito de brindar un conjunto de herramientas para la investigación cuantitativa cienciométrica y bibliométrica. La complejidad de la producción científica se aprecia, sobre todo, gracias a la existencia de los distintos canales de comunicación; no todos los artículos se publican en revistas de acceso abierto (Van Raan, 2014), sin embargo, este recurso nos aproxima a una realidad en construcción.

En los últimos años, este tipo de estudios ha despertado el interés de investigadores que analizan la evolución y tendencias de tópicos más desarrollados (BermeoGiraldo, Acevedo, Palacios, Benjumea y Arango-Botero, 2020; Ramos-Sanz, 2019; Manterola, Astudillo, Arias y Claros, 2013; entre otros). No obstante, y a pesar de que existen estas revisiones, no se ha realizado un estudio que muestre la relación de los temas en cuestión con la creación de grupos de investigación y el perfil de los investigadores.

Como informa Blakeman (2018), la bibliometría ofrece una gama de técnicas y medidas cuantitativas que son usadas para medir la cantidad de publicaciones de un autor, grupos de investigación o instituciones enteras. Junto a ello, se emplean, como herramientas de búsqueda para identificar investigaciones actualizadas, redes de autores y conexiones entre instituciones (Blakeman, 2018). Si bien la bibliometría y las revisiones de literatura son técnicas que ofrece información cuantitativa sintetizada de los artículos publicados, pueden carecer de rigor y presentar errores producidos por el sesgo de los investigadores, por lo tanto, es necesario determinar procesos metodológicos que conduzcan la investigación por caminos más objetivos (Snyder, 2019).

En el caso específico de la arquitectura y la edificación, los estudios bibliométricos de focos de investigación generales son escasos. Ramos-Sanz (2019), por ejemplo, señala que, en el análisis bibliométrico de cinco revistas internacionales de los últimos veinte años, los tópicos más tratados son: a) Transformación visual del $2 \mathrm{~d}$ al $5 \mathrm{~d}$; b) Transformación del objeto arquitectónico en flujos; y c) Transformación del proceso constructivo en información. De acuerdo con Wen, Ren, Lu y Wu (2021), las temáticas tratadas giran alrededor de las tecnologías digitales BIM, herramienta que estaría produciendo los cambios más importantes en la arquitectura y construcción. 


\section{METODOLOGÍA}

La metodología empleada en esta propuesta busca proporcionar una visión descriptiva de lo que se está investigando en el campo de arquitectura en los últimos cinco años, a partir de técnicas de revisión sistemáticas, del uso de herramientas bibliométricas y del monitoreo del interés investigativo de los docentes de la Escuela de Arquitectura (UIDE) que faciliten la identificación de las tendencias de investigación y sus perspectivas. Los pasos para dicha exploración se determinan con base en los tipos de revisiones comunes que consideran un marco de trabajo que, a su vez, implica: 1) búsqueda; 2) evaluación; 3) síntesis; 4) análisis, asociado a la metodología conocida en inglés como "SALSA framework" (Grant y Booth, 2009); y 5) monitoreo de datos bibliográficos con los intereses y perfiles docentes.

En suma, el estudio se enmarca en las tipologías de revisión de literatura y revisión cartográfica definidas por Grant y Booth (2009), puesto que trabajan con documentos publicados que han sido revisados por pares, con criterios de búsqueda con los cuales se puede graficar la información para la consolidación, análisis y relación de la misma, como también por su contribución respecto a la investigación en temáticas de arquitectura y conformación de grupos de investigación. A partir de lo anterior, se sintetizan las características tomadas de las revisiones que guían el trabajo (Tabla1).

La investigación se realiza a partir de un estudio bibliométrico de la producción científica asociado al tema de arquitectura en la base de datos SCOPUS que indexa más de 41000 revistas científicas y que, gracias a su amplia base de datos y su factor de impacto, ha ganado prestigio constituyéndose en un portal de ciencia (Aguaded, 2020). Para definir la ecuación de búsqueda, se emplea la clasificación de códigos ASJC (All Science Journal Classification) de SCOPUS, los cuales permiten limitar la búsqueda dentro de la categoría y clasificación del área temática de arquitectura (2216, architecture), filtrando los resultados.

Para afinar la búsqueda, se aplican los siguientes criterios de inclusión: últimos cinco años, países iberoamericanos - para considerar todos los países que hablan español, pero también a Brasil y Portugal-, y tipos de documentos relacionados (Article-ar / Abstract Report-ab / Book-bk / Book Chapter-ch / Conference Paper-cp / Conference Review-cr / Review-re). Como criterio de exclusión, se limita la indagación a las áreas temáticas asociadas a urbanismo e ingeniería, por cuanto se busca definir un grupo enfocado en arquitectura y edificación. De esta manera, se obtienen 1465 documentos, que son exportados en formato bib y csv, incluyendo citas, información bibliográfica, resúmenes y palabras clave, lo cual permite tener una visión clara del ámbito de estudio (Tabla 2).

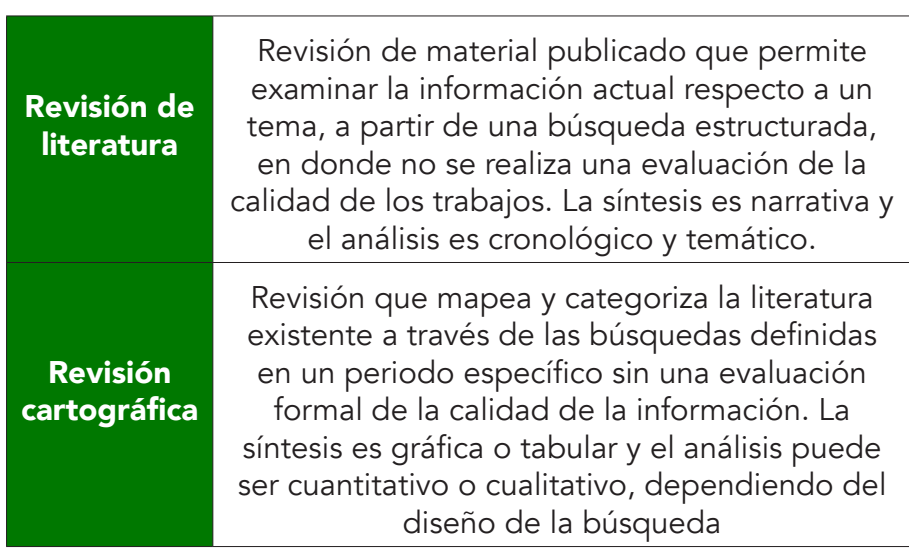

Tabla 1. Síntesis de las tipologías de revisiones empleadas en la investigación Fuente: Elaboración de los autores con base en Grant y Booth (2009)

\begin{tabular}{|c|c|c|c|c|c|}
\hline $\begin{array}{l}\text { Ecuación } \\
\text { de } \\
\text { búsqueda }\end{array}$ & $\begin{array}{c}\text { Base } \\
\text { de } \\
\text { datos }\end{array}$ & Años & Tipos de doc & Países & $\begin{array}{c}\text { Doc. } \\
\text { analizados }\end{array}$ \\
\hline 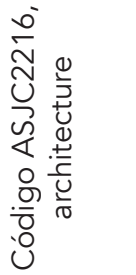 & $\begin{array}{l}\qquad \\
\supset \\
0 \\
0 \\
\sim\end{array}$ & 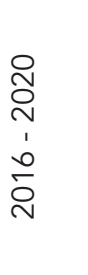 & 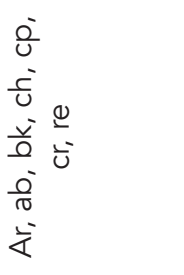 & 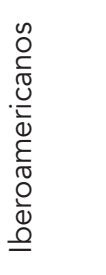 & 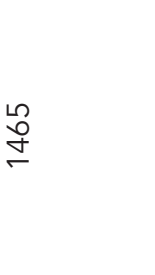 \\
\hline
\end{tabular}

Tabla 2. Estrategias de búsqueda. Fuente: Elaboración de los autores.

Para la evaluación, se utiliza la herramienta Bibliometrix, que es de código abierto y facilita el mapeo científico (Duque y Cervantes, 2019; Aria y Cucurrullo, 2017). Esta herramienta analiza la información a través de indicadores de producción, visibilidad e impacto y de colaboración (Chuquin y Salazar, 2019). La información se muestra mediante cartografías científicas y datos, lo que hace posible realizar síntesis a través del estudio de datos respecto a producción científica anual, productividad por países, impacto en revistas, palabras clave, tendencia de tópicos, entre otros factores. Los datos se extraen como gráficos y tablas que permiten clasificar y relacionar la información. En la fase siguiente se realiza el análisis descriptivo bibliométrico, examinando a profundidad la información para identificar los temas relevantes que se investigan actualmente. Finalmente, esta información se contrasta con los resultados obtenidos de la encuesta aplicada a los docentes de la Escuela de Arquitectura (UIDE) para, con ello, generar los resultados y la discusión que identifican las relaciones de los temas con el perfil e interés de los investigadores (Figura 1).

Los indicadores de Bibliometrix (Gallardo, 2016) se clasifican en tres tipos: el primero se enfoca en indicadores de producción científica; el segundo agrupa los indicadores de impacto y visibilidad del autor; y el último contiene indicadores de colaboración. Los 

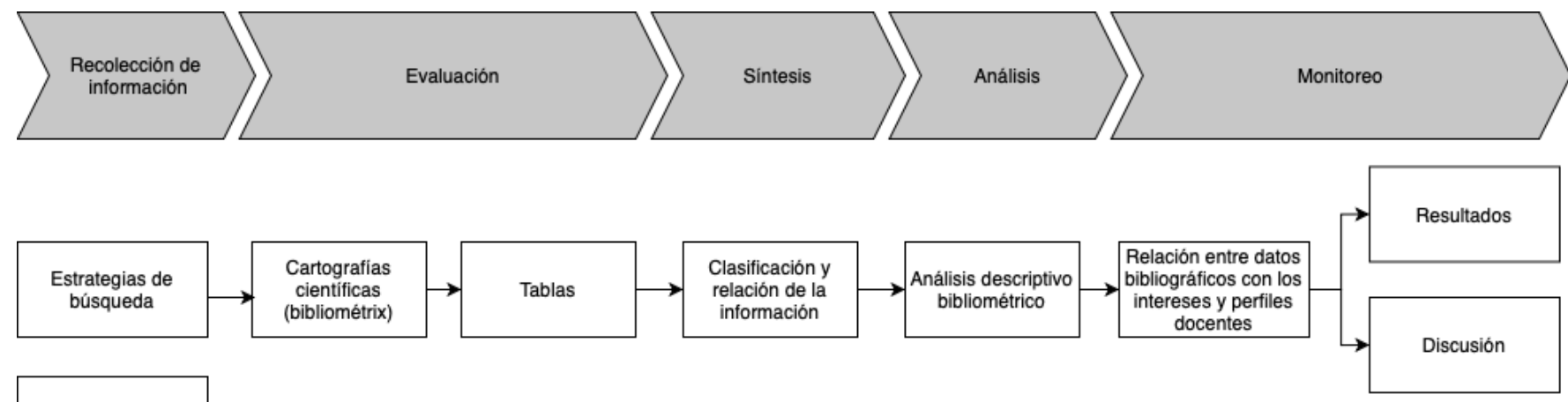

Extracción de datos .bib, .csv

Figura 1. Procedimiento metodológico. Fuente: Elaboración de los autores con base en Grant y Booth (2009).

\begin{tabular}{|c|c|c|c|c|}
\hline $\begin{array}{l}\text { TIPOS DE INDICADORES } \\
\text { EN BIBLIOMETRIX }\end{array}$ & INDICADOR & $\begin{array}{l}\text { ESTRATEGIA DE } \\
\text { ANÁLISIS }\end{array}$ & OBJETIVO DE ANÁLISIS & RESULTADOS \\
\hline Productividad & $\begin{array}{l}\text { Índice } \mathrm{H} \text { en } \\
\text { revistas }\end{array}$ & $\begin{array}{l}\text { Describir } \\
\text { Comparar }\end{array}$ & $\begin{array}{l}\text { Índice H: descripción } \\
\text { y comparación de los } \\
\text { artículos en revistas. }\end{array}$ & $\begin{array}{l}\text { Identificar de las revistas de } \\
\text { mayor producción científica } \\
\text { en la temática investigada. }\end{array}$ \\
\hline Impacto y visibilidad & $\begin{array}{l}\text { Ley de Lotka } \\
\text { Relevancia de las } \\
\text { afiliaciones } \\
\text { Relación autores } \\
\text { por países } \\
\text { Producción } \\
\text { científica por país }\end{array}$ & $\begin{array}{l}\text { Describir } \\
\text { Identificar } \\
\text { Relacionar }\end{array}$ & $\begin{array}{l}\text { Ley de Lotka: Identificar si } \\
\text { la mayoría de los autores } \\
\text { publican el menor número } \\
\text { de trabajos, mientras } \\
\text { que unos pocos autores } \\
\text { publican la mayor parte } \\
\text { de la bibliografía relevante } \\
\text { sobre un tema de } \\
\text { investigación, y forman el } \\
\text { grupo más prolífico. } \\
\text { Relevancia de las } \\
\text { afiliaciones: Identificación } \\
\text { de las instituciones y } \\
\text { universidades a las que } \\
\text { pertenece el autor. } \\
\text { Relación de autores por } \\
\text { países: Identificación } \\
\text { de los países a los que } \\
\text { pertenecen los autores que } \\
\text { más publican. } \\
\text { Producción científica } \\
\text { por país: Identificación } \\
\text { de los países con mayor } \\
\text { producción científica en los } \\
\text { temas de análisis en base } \\
\text { a los autores de mayor } \\
\text { impacto. } \\
\text { Países más citados: } \\
\text { Identificación de los países } \\
\text { con mayor producción } \\
\text { científica en los temas } \\
\text { de análisis en base a los } \\
\text { autores de mayor impacto. }\end{array}$ & $\begin{array}{l}\text { Identificar a los autores, } \\
\text { las afiliaciones, los países } \\
\text { y la producción científica } \\
\text { relevante que ayudan a } \\
\text { relacionar datos. }\end{array}$ \\
\hline
\end{tabular}




\begin{tabular}{|c|c|c|c|c|}
\hline Colaboración & $\begin{array}{l}\text { Documento más } \\
\text { citado por año } \\
\begin{array}{c}\text { Palabras clave de } \\
\text { autor }\end{array} \\
\text { Estructura } \\
\text { conceptual } \\
\text { Estructura social }\end{array}$ & $\begin{array}{l}\text { Identificar } \\
\text { Relacionar } \\
\text { Comparar } \\
\text { Conectar }\end{array}$ & $\begin{array}{l}\text { Documento más citado } \\
\text { por año: Identificar los } \\
\text { documentos más citados } \\
\text { que se relacionan con la } \\
\text { temática en estudio. } \\
\text { Palabras clave de autor: } \\
\text { Relaciona las palabras más } \\
\text { frecuentes. } \\
\text { Estructura conceptual: } \\
\text { Identificación de la } \\
\text { conexión entre los } \\
\text { términos de selección. } \\
\text { Estructura social: Relación } \\
\text { entre autores, países e } \\
\text { instituciones. }\end{array}$ & $\begin{array}{l}\text { Identificar a través de las } \\
\text { palabras clave los temas en } \\
\text { tendencia de publicación en } \\
\text { la temática. } \\
\text { Relacionar y analizar la } \\
\text { conexión de los términos } \\
\text { seleccionados. } \\
\text { Identificar los autores, países } \\
\text { e instituciones que se vinculan } \\
\text { en la temática. }\end{array}$ \\
\hline
\end{tabular}

Tabla 3. Tipos de indicadores en Bibliometrix. Fuente: Elaboración de los autores con datos de la guía (Ciencia Unisalle, 2020).

indicadores de productividad científica registran el crecimiento y distribución de la producción científica por años, la concentración de los temas en las revistas y la distribución geográfica de la producción. Los indicadores de impacto y visibilidad revelan la influencia que produce el contenido en la comunidad científica. Por último, los indicadores de colaboración pueden leerse a través de mapas estructurales que relacionan varios indicadores en una sola imagen.

En el marco del presente estudio, se seleccionaron los indicadores capaces de conducir a los hallazgos requeridos para sustentar el objetivo de la investigación (Tabla 3).

Como última instancia fue llevada a cabo una encuesta analítica de preguntas cerradas a los docentes de la Escuela de Arquitectura (UIDE), mediante la cual se obtuvo información referente a: 1) título de tercer nivel; 2) título de cuarto nivel; y 3) preferencias de investigación según los datos bibliométricos. Esto permitió relacionar el perfil de los docentes con los temas de interés principales que podrán ser tratados en el grupo de investigación.

\section{RESULTADOS Y DISCUSIÓN}

Previamente se describieron las características claves del proceso de investigación, en donde limitar las búsquedas y enfocar la revisión dentro del dominio de "Arquitectura" permitió identificar temáticas relevantes de actualidad que contribuyeran a enfocar el trabajo de los grupos de investigación en arquitectura y el establecimiento de las líneas específicas de investigación.

A partir de lo anterior, se obtuvieron una serie de resultados que se exponen a continuación. Respecto a la producción

\begin{tabular}{|c|c|}
\hline Tipos de Documentos & Resultados \\
\hline Artículos & 998 \\
\hline Capítulos de libro & 64 \\
\hline Artículos de conferencia & 327 \\
\hline Revisiones de literatura & 76 \\
\hline
\end{tabular}

Tabla 4. Tipos de documentos encontrados. Fuente: Elaboración de los autores con Bibliometrix.

científica de los últimos 5 años y considerando los 1465 documentos exportados de la búsqueda, circunscritos al ámbito de arquitectura, los documentos publicados son los referidos en la Tabla 4.

Con base en una revisión general de los indicadores generados en Bibliometrix y luego de seleccionar a través de criterios cualitativos los indicadores que abordan el objeto de la investigación, se ejecutó en el programa la ecuación de búsqueda con los códigos expuestos en el apartado dedicado a la metodología.

El índice $\mathrm{H}$ en revistas evidencia que, para la presente investigación, las tres revistas de mayor relevancia en las que se advierte mayor producción de artículos son Journal of building Engimeering, ARQ y International Journal of Architectural Heritage. De los 1465 documentos publicados la primera revista corresponde a un $7.78 \%$, la segunda, a un $6.69 \%$, y la tercera, a un $6.01 \%$; porcentajes que sumados constituyen el $20.68 \%$ de la producción total. Las diez revistas con mayor productividad se aprecian en la Tabla 5.

Delas diez revistasidentificadas, se observa que cinco publican frecuentemente artículos en español: cuatro universidades de Chile y una de España. Una vez identificadas las revistas, 


\begin{tabular}{|c|c|c|c|c|}
\hline Revistas & Documentos & Idioma de la publicación & $\begin{array}{c}\text { Afiliación Universitaria } \\
\text { Directa }\end{array}$ & País Sede \\
\hline Journal of Building Engineering & 114 & Inglés & No & Internacional \\
\hline Arq & 101 & Español & Sí & Chile \\
\hline $\begin{array}{c}\text { International Journal of Architectural } \\
\text { Heritage }\end{array}$ & 88 & Inglés & No & Internacional \\
\hline Aus & 86 & Español & Sí & Chile \\
\hline Revista 180 & 83 & Español & Sí & Chile \\
\hline Revista Invi & 72 & Español & Sí & Chile \\
\hline Structures & 59 & Inglés & No & Internacional \\
\hline $\begin{array}{l}\text { Advances In Science Technology and } \\
\text { Innovation }\end{array}$ & 54 & Inglés & No & Internacional \\
\hline Buildings & 45 & Inglés & No & Internacional \\
\hline Architecture City and Environment & 43 & Español & Sí & España \\
\hline
\end{tabular}

Tabla 5. Índice H. Fuente: Elaboración de los autores con Bibliometrix.

\begin{tabular}{|c|c|c|}
\hline Documentos escritos & Nro. de autores & Proporción de autores \\
\hline 1 & 2702 & 0,829 \\
\hline 2 & 393 & 0,121 \\
\hline 3 & 97 & 0,03 \\
\hline 4 & 29 & 0,009 \\
\hline 5 & 17 & 0,005 \\
\hline 6 & 4 & 0,001 \\
\hline 7 & 5 & 0,002 \\
\hline 8 & 4 & 0,001 \\
\hline 9 & 2 & 0,001 \\
\hline 10 & 3 & 0,001 \\
\hline 11 & 1 & 0 \\
\hline 12 & 1 & 0 \\
\hline 17 & 1 & 0 \\
\hline 25 & 1 & 0 \\
\hline 39 & 1 & 0 \\
\hline
\end{tabular}

Tabla 6. Ley de Lotka. Fuente: Elaboración de los autores con Bibliometrix.

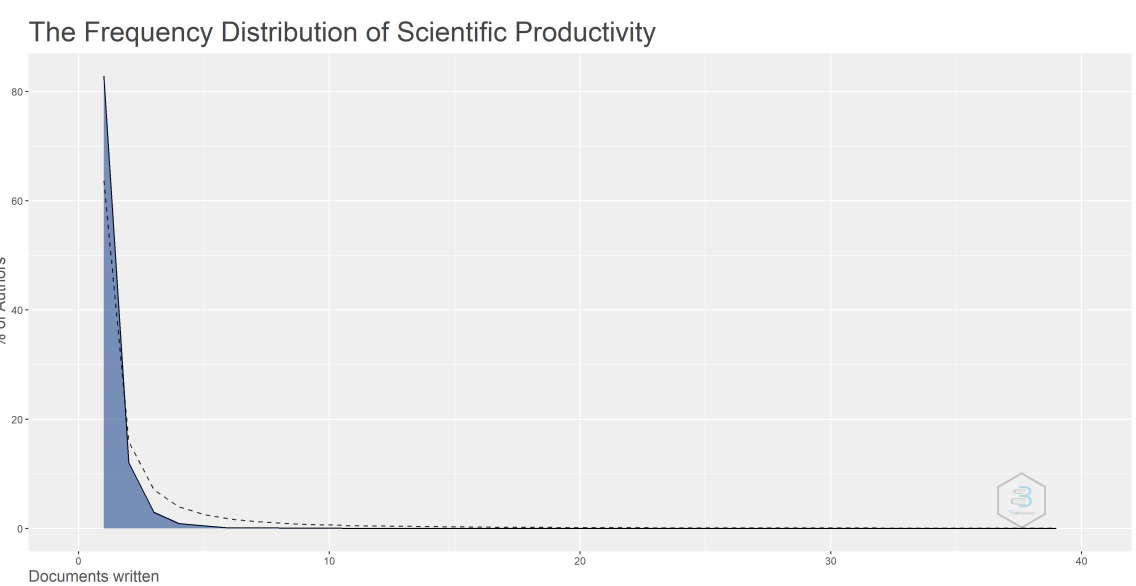

Figura 2. Ley de Lotka. Fuente: Elaboración de los autores con Bibliometrix. 


\begin{tabular}{|c|c|c|}
\hline Afiliación & Documentos & País \\
\hline Pontificia Universidad Católica de Chile & 148 & Chile \\
\hline Universidad de Lisboa & 120 & Portugal \\
\hline Universidad de Minho & 90 & Portugal \\
\hline Universidad de Chile & 84 & Portugal \\
\hline Universidad de Porto & 70 & Portugal \\
\hline Universidad de Coimbra & 59 & Portugal \\
\hline Universidad de Aveiro & 42 & Chile \\
\hline Universidad del Bío-Bío & 39 & \\
\hline
\end{tabular}

Tabla 7. Relevancia de las afiliaciones. Fuente: Elaboración de los autores con Bibliometrix.

\section{Country Scientific Production}

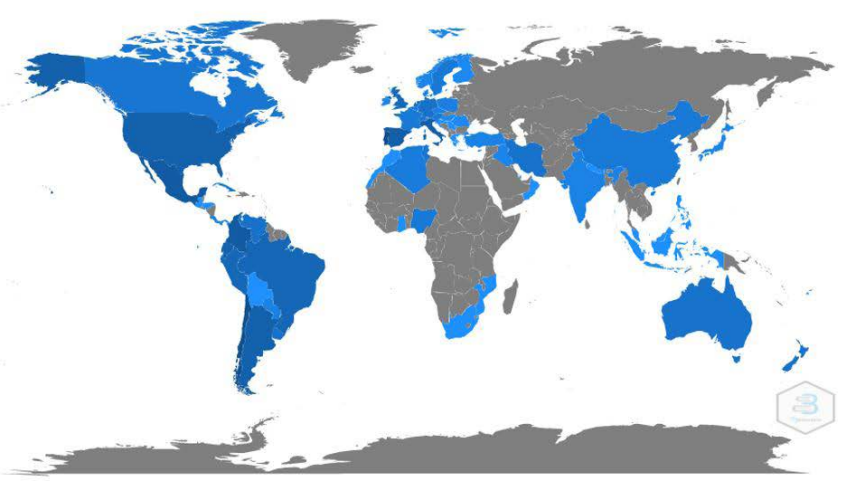

\begin{tabular}{lc}
\hline Países & Documentos \\
Portugal & 861 \\
Chile & 712 \\
Colombia & 213 \\
México & 213 \\
España & 136 \\
Italia & 124 \\
Usa & 110 \\
Argentina & 106 \\
Ecuador & 105 \\
Brasil & 61 \\
Perú & 57
\end{tabular}

Figura 3. Indicador producción científica por países. Fuente: Elaboración de los autores con Bibliometrix.

las mismas se convierten en material principal para la revisión de la producción científica en tendencia.

Según la ley de Lotka, durante el período de este estudio y de acuerdo a la proporción de autores, se vislumbra que sólo el $0.5 \%$ de los autores publican un artículo por año, mientras que el $82.9 \%$ publica aproximadamente un artículo en los 5 años estudiados (Figura 2). Se demuestra así que la curva esperada de la frecuencia de distribución de productividad científica está por debajo de la ley de Lotka.

En cuanto al índice de relevancia de las afiliaciones, surge, en primer lugar, el impacto de la Pontificia Universidad Católica de Chile, con el $5.11 \%$ del total, seguida por la Universidad de Minho, con el 3.10\% y, en tercer lugar, la Universidad de Chile con el $2.90 \%$. Las tres instituciones representan el $11.11 \%$ de las afiliaciones correspondientes a los artículos publicados. En la siguiente tabla se pueden apreciar las 10 primeras universidades que, para la ecuación de búsqueda, presentan mayor impacto y visibilidad. (Tabla 7).

Cabe agregar que aparecen en este índice otras universidades de Colombia, Chile, Portugal y España, como la Universidad Nova de Lisboa, la Universidad Nacional de Colombia, la Universidad Diego Portales, la Universidad Politécnica de Madrid, entre otras. Asimismo, del total de 1219 universidades analizadas aquí, 870 registra un artículo publicado, es decir, el $71 \%$ de las publicaciones.

Las principales plataformas de comunicación que usan los autores dentro del ámbito de arquitectura se encuentran en Chile y Portugal, lo cual pone de manifiesto que las universidades identificadas son fuente de información en cuanto a su estructura de publicación y temas de interés.

El análisis del indicador de la producción científica (Figura 3) revela la capacidad académica de los países. En concreto, el país con mayor producción científica es Portugal, con un total de 861 documentos publicados en los últimos 5 años, al que le siguen Chile, Colombia y México. Por su parte, el indicador de la relación autores por países (Figura 4) expresa la relación del MCP (Multiple Countries Publication) y SCP (Single Country Publication), que permite identificar que Portugal cuenta con mayor MCP: de los 296 artículos publicados, 55 cuentan al menos con un coautor de un país diferente. Italia, en tanto, es el segundo país con mayor MCP: de los 32 artículos publicados todos poseen colaboración internacional. 
Tendencias de investigación en arquitectura entre 2016-2020 en la base de datos SCOPUS y su relación con la creación de grupos de investigación Claudia Costa-De los Reyes, Silvia Viñán-Ludeña, María Isabel Vivanco-Villavicencio, Fernando Moncayo-Serrano

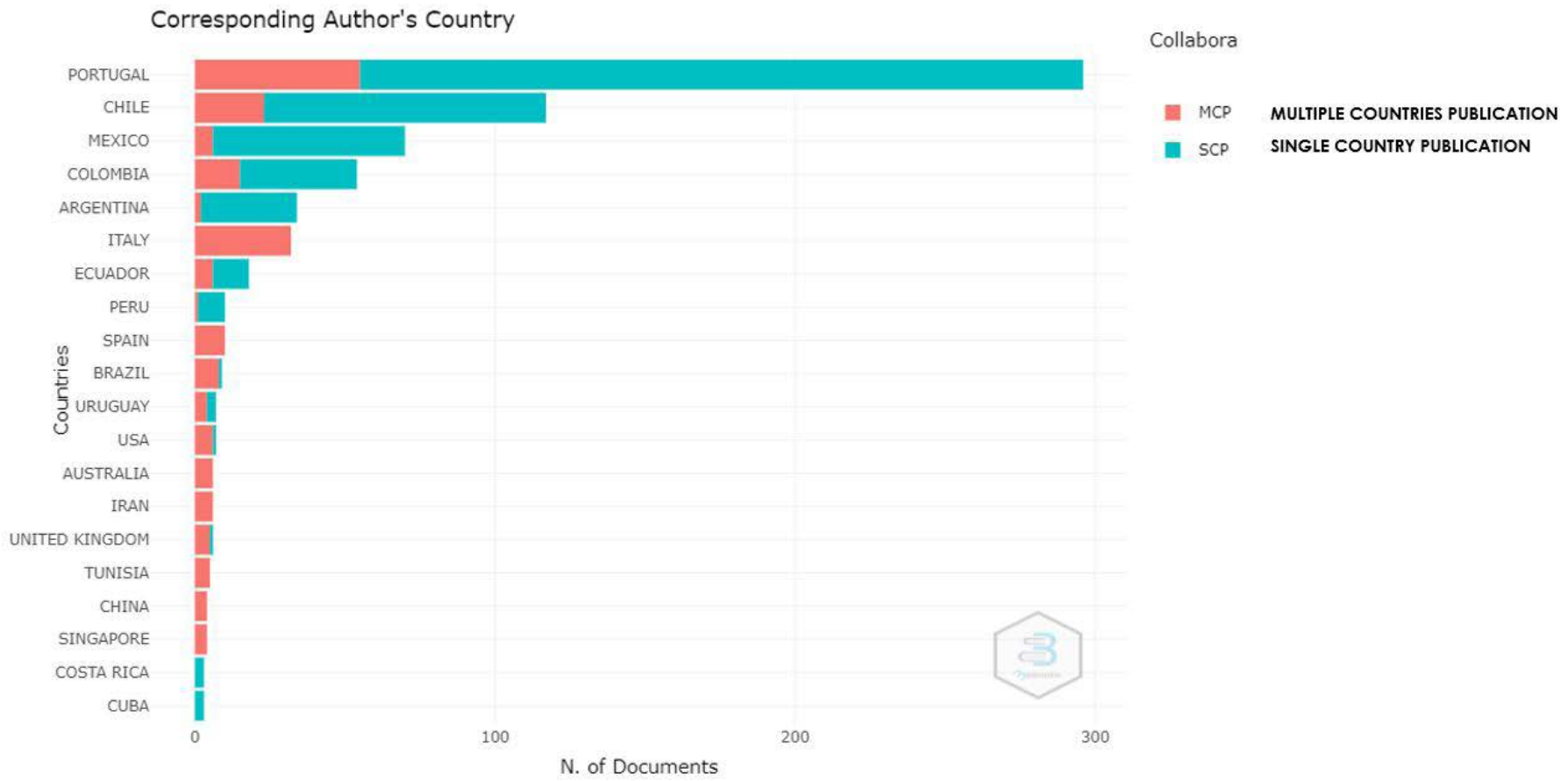

Figura 4. Indicador de la relación autores por países. Fuente: Elaboración de los autores con Bibliometrix.

\section{Most Cited Contries}

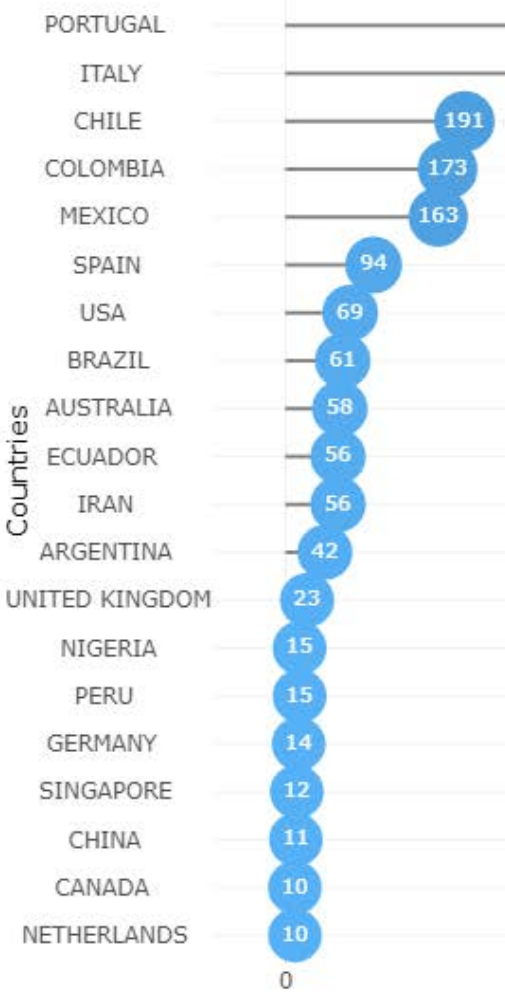

\section{(19)}




\section{Average Article Citations per Year}

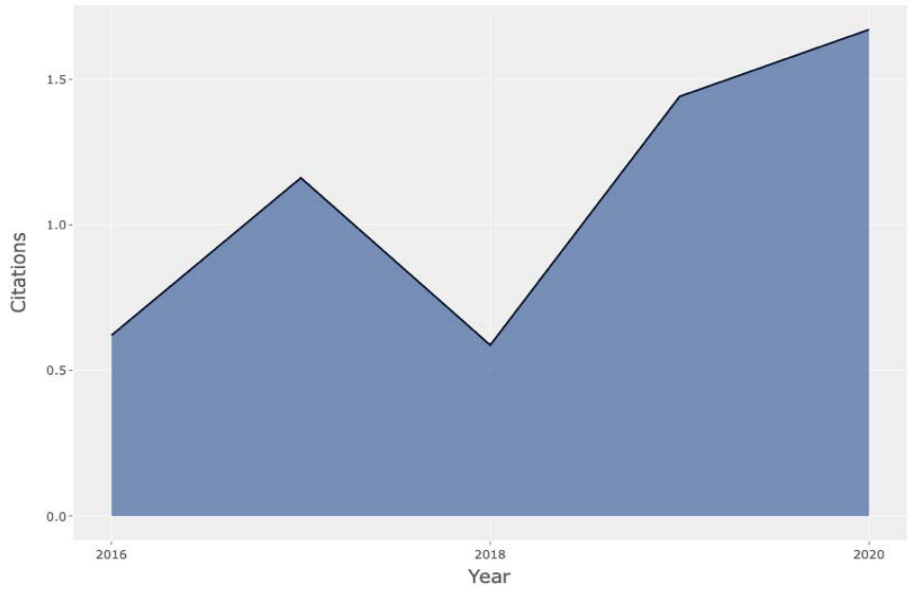

Figura 6. Promedio de citas de artículos por año. Fuente: Elaboración de los autores con Bibliometrix.

Con el indicador de los países más citados (Figura 5), es posible distinguir que Portugal está en la vanguardia de la producción científica y, por ende, es el país con mayor número de citaciones: en los últimos 5 años obtuvo 1551 citaciones. Pero es importante constatar que es relevante contar con la colaboración internacional, pues se evidencia que Italia, Portugal y Chile poseen mayor número de citaciones en sus artículos.

Tomando en cuenta el indicador de colaboración, respecto al documento más citado por año (Figura 6), se nota una relación con el código definido para la búsqueda asociado a "arquitectura" y a los criterios de exclusión: uno o más documentos publicados en 2020 recogen el mayor número de citas promedio por año, lo cual implica que el tema tiene incidencia de búsquedas.

De acuerdo a la estructura conceptual en la que se realiza la identificación de la conexión entre los temas o tópicos filtrado, y según el campo de palabras clave de autor y la concurrencia de las mismas, se agrupan en nodos principales los temas y se establecen subgrupos que permiten determinar las tendencias de investigación en arquitectura en los últimos 5 años.

En función de los datos obtenidos a través del mapa temático en Bibliometrix, la tendencia de temas relacionados con las palabras clave de autor encontradas que se identifican en los documentos, se puede agrupar bajo tres términos en dichos documentos contenidos: diseño, construcción y sustentabilidad (Tabla 8). Esta información es filtrada y analizada según los criterios de búsqueda antes planteados, con el objetivo de clasificar la misma en contenedores principales que permitan identificar los perfiles necesarios para abordar los tópicos en tendencia.

Gracias a la cartografía de dinámica de palabras (Figura 7), se puede conocer, a través de las palabras clave, cómo los temas en tendencia de publicación en arquitectura se han

\begin{tabular}{|c|c|c|}
\hline Palabras & $\begin{array}{l}\text { Etiqueta del } \\
\text { grupo }\end{array}$ & $\begin{array}{l}\text { Relación con los perfiles } \\
\text { de investigador }\end{array}$ \\
\hline Ensayo & Proyecto & Construcción \\
\hline Leyes & Proyecto & Construcción \\
\hline Madera & Proyecto & Construcción \\
\hline Patrimonio & Patrimonio & Construcción \\
\hline Rehabilitación & Rehabilitación & Construcción \\
\hline $\begin{array}{l}\text { Propiedades } \\
\text { mecánicas }\end{array}$ & Rehabilitación & Construcción \\
\hline $\begin{array}{l}\text { Esfuerzo a la } \\
\text { compresión }\end{array}$ & Rehabilitación & Construcción \\
\hline $\begin{array}{l}\text { Concreto } \\
\text { autocompactante }\end{array}$ & Rehabilitación & Construcción \\
\hline Albañilería & Albañilería & Construcción \\
\hline $\begin{array}{l}\text { Concreto } \\
\text { reforzado }\end{array}$ & Albañilería & Construcción \\
\hline $\begin{array}{l}\text { Evaluación } \\
\text { sísmica }\end{array}$ & Albañilería & Construcción \\
\hline $\begin{array}{l}\text { Vulnerabilidad } \\
\text { sísmica }\end{array}$ & Albañilería & Construcción \\
\hline $\begin{array}{l}\text { Patrimonio } \\
\text { cultural }\end{array}$ & Albañilería & Construcción \\
\hline $\begin{array}{l}\text { Modelado } \\
\text { numérico }\end{array}$ & Albañilería & Construcción \\
\hline $\begin{array}{l}\text { Análisis de } \\
\text { presión }\end{array}$ & Albañilería & Construcción \\
\hline Proyecto & Proyecto & Diseño arquitectónico \\
\hline Diseño & Proyecto & Diseño arquitectónico \\
\hline Edificación & Proyecto & Diseño arquitectónico \\
\hline Construcción & Proyecto & Diseño arquitectónico \\
\hline Concreto & Proyecto & Diseño arquitectónico \\
\hline Coexistencia & Proyecto & Diseño arquitectónico \\
\hline Alojamiento & Alojamiento & Diseño arquitectónico \\
\hline $\begin{array}{l}\text { Arquitectura } \\
\text { moderna }\end{array}$ & Alojamiento & Diseño arquitectónico \\
\hline Diseño urbano & Alojamiento & Diseño arquitectónico \\
\hline Vivienda social & Vivienda social & Diseño arquitectónico \\
\hline Sostenibilidad & Sustentabilidad & Sustentabilidad \\
\hline Historia & Construcción & Sustentabilidad \\
\hline $\begin{array}{l}\text { Eficiencia } \\
\text { energética }\end{array}$ & $\begin{array}{l}\text { Eficiencia } \\
\text { energética }\end{array}$ & Sustentabilidad \\
\hline Cambio climático & $\begin{array}{l}\text { Eficiencia } \\
\text { energética }\end{array}$ & Sustentabilidad \\
\hline Confort térmico & Vivienda social & Sustentabilidad \\
\hline Estabilidad & Vivienda social & Sustentabilidad \\
\hline
\end{tabular}

Tabla 8. Clasificación de palabras clave. Fuente: Elaboración de los autores. 


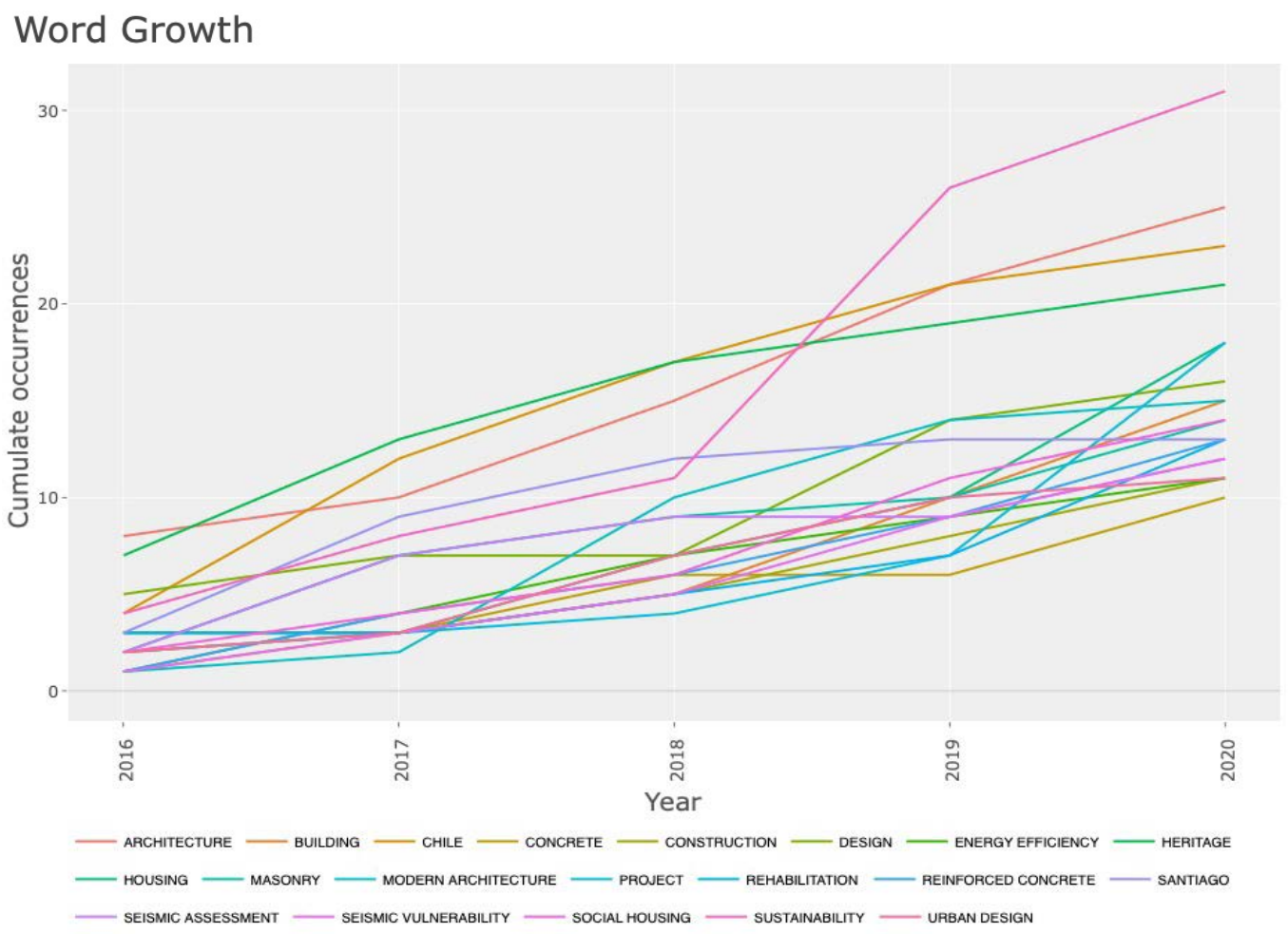

Figura 7. Crecimiento de la tendencia de palabras. Fuente: Elaboración de los autores con Bibliometrix.

posesionado con el tiempo. En este caso, queda expuesto que la sustentabilidad en los últimos 5 años es una temática recurrente y que alcanza su pico más alto en el año 2020, con una clara ventaja frente a los otros tópicos en tendencia.

Desde otro punto de vista, los rangos de centralidad y densidad informan acerca de las asociaciones externas e internas de los grupos. En el rango de centralidad se observa que los grupos de sustentabilidad, proyecto y alojamiento tienden a ser vinculantes y pueden ser abordados de forma multidisciplinar; en cambio, los temas relativos a patrimonio, albañilería y eficiencia energética presentan mayor frecuencia de asociaciones internas.

A través del análisis de la estructura social, se revisa la vinculación entre autores, universidades y países dentro de la investigación en arquitectura, lo que permite buscar alianzas para la colaboración en investigaciones y la creación de redes entre grupos de investigación.

De 31 universidades identificadas que investigan las temáticas ligadas a arquitectura, la Pontificia Universidad Católica de Chile y la University of Minho, son aquellas con mayor intermediación entre la Red de Colaboración de Universidades. Con la primera colaboran aproximadamente 15 universidades y con la segunda, 12 universidades. Sin embargo, no se muestra una conexión de trabajo entre ellas. En la Figura 8, se puede ver, además, redes más pequeñas de colaboración entre la Pontifica Universidad Javeriana, la Universidad de Colombia y la Universidad de los Andes y una red de colaboración entre la Universidad de Cuenca, Universidad del Azuay y la Universidad Alberto Hurtado,

\begin{tabular}{|c|c|c|}
\hline Grupo & $\begin{array}{c}\text { Rango de } \\
\text { centralidad }\end{array}$ & $\begin{array}{c}\text { Rango de } \\
\text { densidad }\end{array}$ \\
\hline Proyecto & 12 & 7 \\
\hline Patrimonio & 8 & 12 \\
\hline Rehabilitación & 10 & 8 \\
\hline México & 6 & 6 \\
\hline Albañilería & 9 & 10 \\
\hline Sustentabilidad & 13 & 3 \\
\hline Alojamiento & 11 & 2 \\
\hline Eficiencia energética & 7 & 9 \\
\hline Vivienda social & 5 & 5 \\
\hline
\end{tabular}

Tabla 9. Rangos de centralidad y densidad de los temas de tendencia. Fuente: Elaboración de los autores con Bibliometrix.

vinculada a la red de la Pontificia Universidad Católica de Chile (Figura 8).

Asimismo, se patentiza que, a pesar de que existe colaboración entre autores dentro de las investigaciones, esta es mínima, pues solamente se visualizan ocho redes de autores entre todos los documentos analizados. Ello refleja que, en los últimos años, para consolidar las investigaciones dentro de la temática de arquitectura, estas se llevan a cabo de forma aislada, dejando de lado la creación de redes y grupos de investigación (Figura 9). 

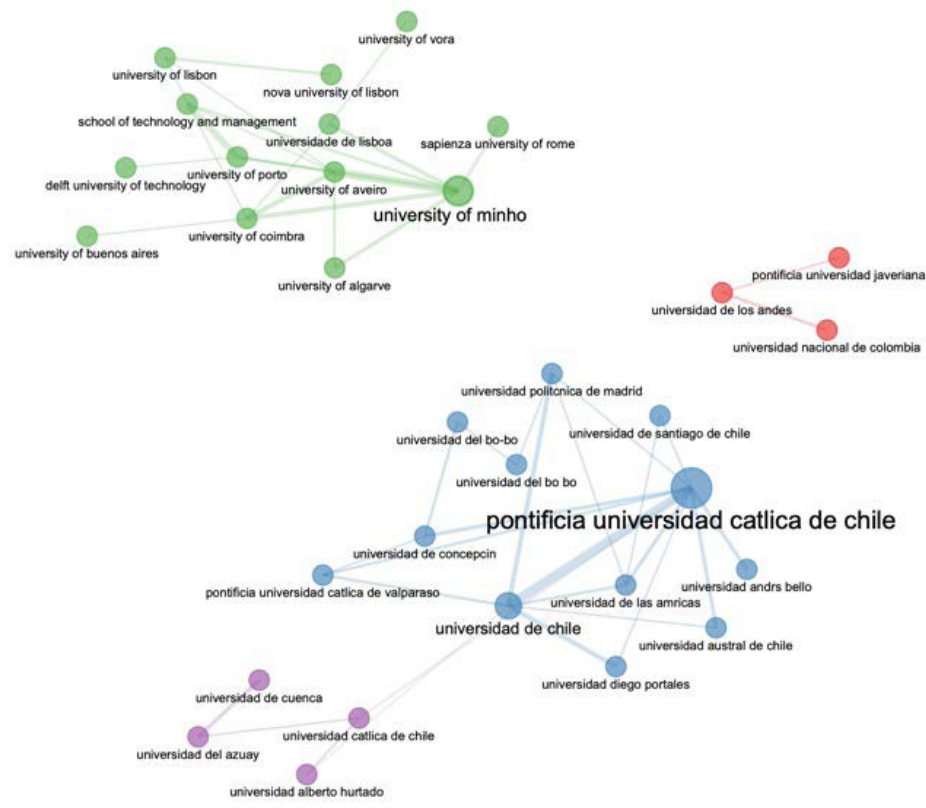

Figura 8. Red de colaboración entre universidades. Fuente: Elaboración de los autores con Bibliometrix.

Por otra parte, relacionando campos específicos entre palabras clave, países y las afiliaciones de los autores, se pueden determinar los temas en tendencia que se investigan durante los últimos cinco años. La Figura 10 muestra que la sustentabilidad constituye el tema más investigado, que es abordado por varias universidades en diferentes países. A este campo le siguen el de diseño, materiales, patrimonio, alojamiento, eficiencia energética, confort, entre otros. Así, es dable identificar, según el tema de investigación, la posibilidad de generar alianzas con otras universidades para la creación de redes y grupos de investigación.

Se aplicó una encuesta a 19 de los 22 docentes de la Escuela (UIDE), para relacionar el perfil de formación (arquitectura y proyectos, construcciones, urbanismo y otros), con los temas encontrados en la bibliometría. En los resultados obtenidos se advirtió que las preferencias de los grupos de perfiles docentes son coincidentes (Figura 11) en arquitectura y diseño arquitectónico; sin embargo, sustentabilidad y eficiencia energética constituyen temas de interés transversal en 3 de los cuatro grupos monitoreados.

Dada la complementariedad investigación-docencia en las universidades, cabe señalar que la tendencia marcada por la temática "sustentabilidad" es visible también en el currículo de la formación profesional de arquitectos. En efecto, González y Trebilcock (2012), por ejemplo, mencionan que en el $76 \%$ de los programas de estudios de Arquitectura en Hispanoamérica se integra dicho concepto. En ese sentido, la literatura académica ha desarrollado la definición de "diseño sustentable" como un proceso creativo que busca la reducción de gastos en los recursos naturales empleados, tales como la contaminación al suelo, aire y agua, también el

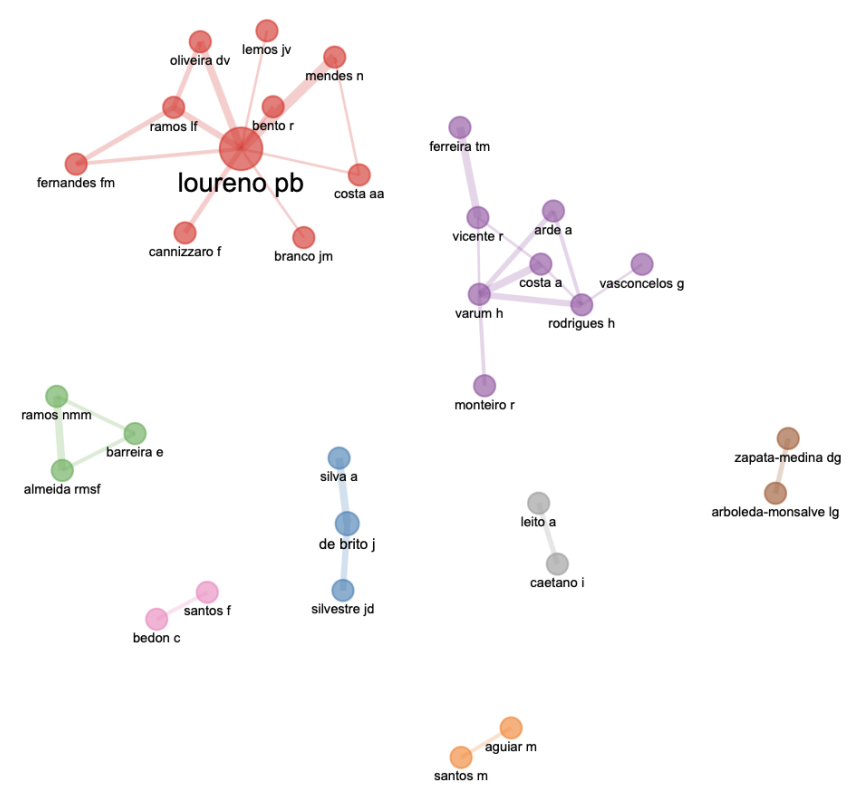

Figura 9. Redes de colaboración de autores. Fuente: Elaboración de los autores con Bibliometrix.

confort al interior de las edificaciones, el ahorro económico y financiero en los proyectos constructivos, así como la reducción de los desperdicios y desechos generados en la construcción (D'Amanzo, Mercado y Ganem-Karlen, 2020).

\section{CONCLUSIONES}

Para la formación de grupos de investigación resulta importante relacionar los enfoques actuales de investigación con los perfiles de los posibles participantes. Desde esa perspectiva, la bibliometría, en términos generales, permite visualizar las universidades de mayor producción científica y los temas actuales de potenciales investigaciones, no obstante, es necesario también articular estos temas con los intereses de investigación de los académicos. Esto ayuda a crear redes internas y externas de colaboración para plantear temas macro con diferentes subtemas, en los cuales los participantes trabajen por un mismo objetivo, pero con diferentes alternativas.

El monitoreo propuesto demuestra que, a pesar de que existan diferentes perfiles de especialización, se pueden determinar temas transversales de interés; para este caso, por ejemplo: la sustentabilidad, la eficiencia energética y el diseño arquitectónico son preferencias recurrentes en los campos de los perfiles docentes.

La aplicación de técnicas bibliométricas en el campo amplio de la arquitectura es escasa por lo que la búsqueda de nichos de investigación resulta difícil. Sin embargo, actualmente la bibliometría está siendo ampliamente 
Tendencias de investigación en arquitectura entre 2016-2020 en la base de datos SCOPUS y su relación con la creación de grupos de investigación Claudia Costa-De los Reyes, Silvia Viñán-Ludeña, María Isabel Vivanco-Villavicencio, Fernando Moncayo-Serrano

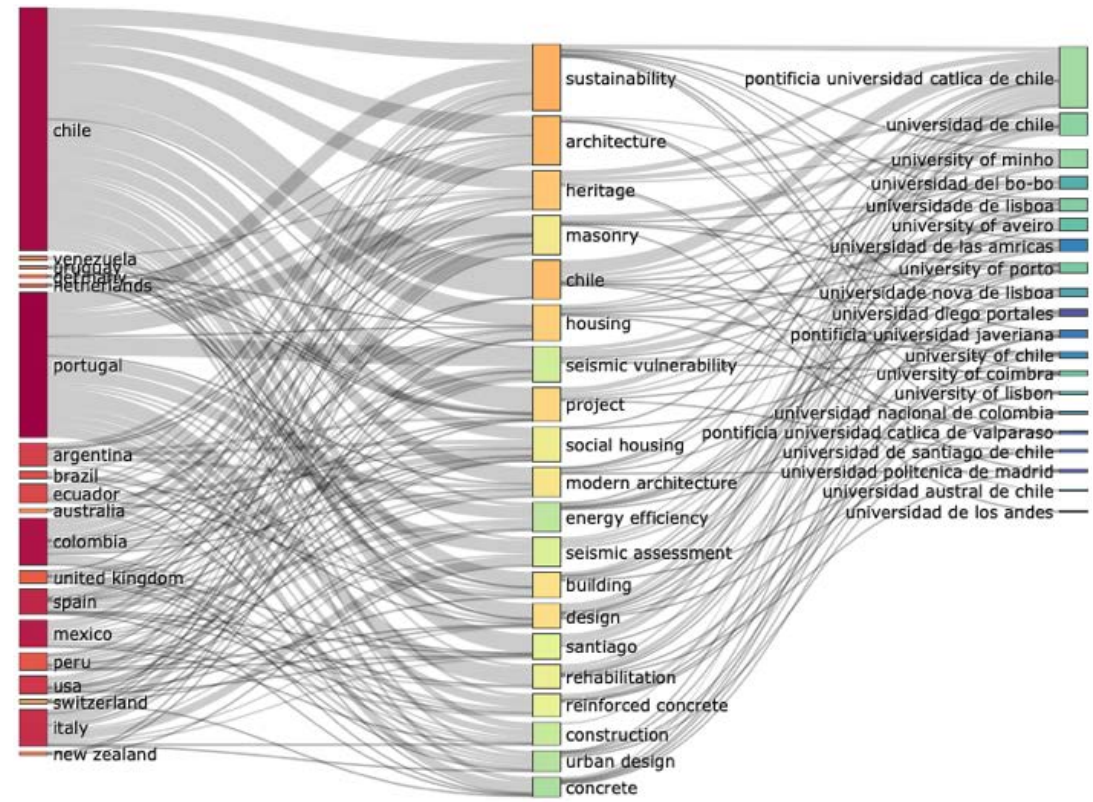

Figura 10. Relación entre temas, países y afiliaciones. Fuente: Elaboración de los autores con Bibliometrix.
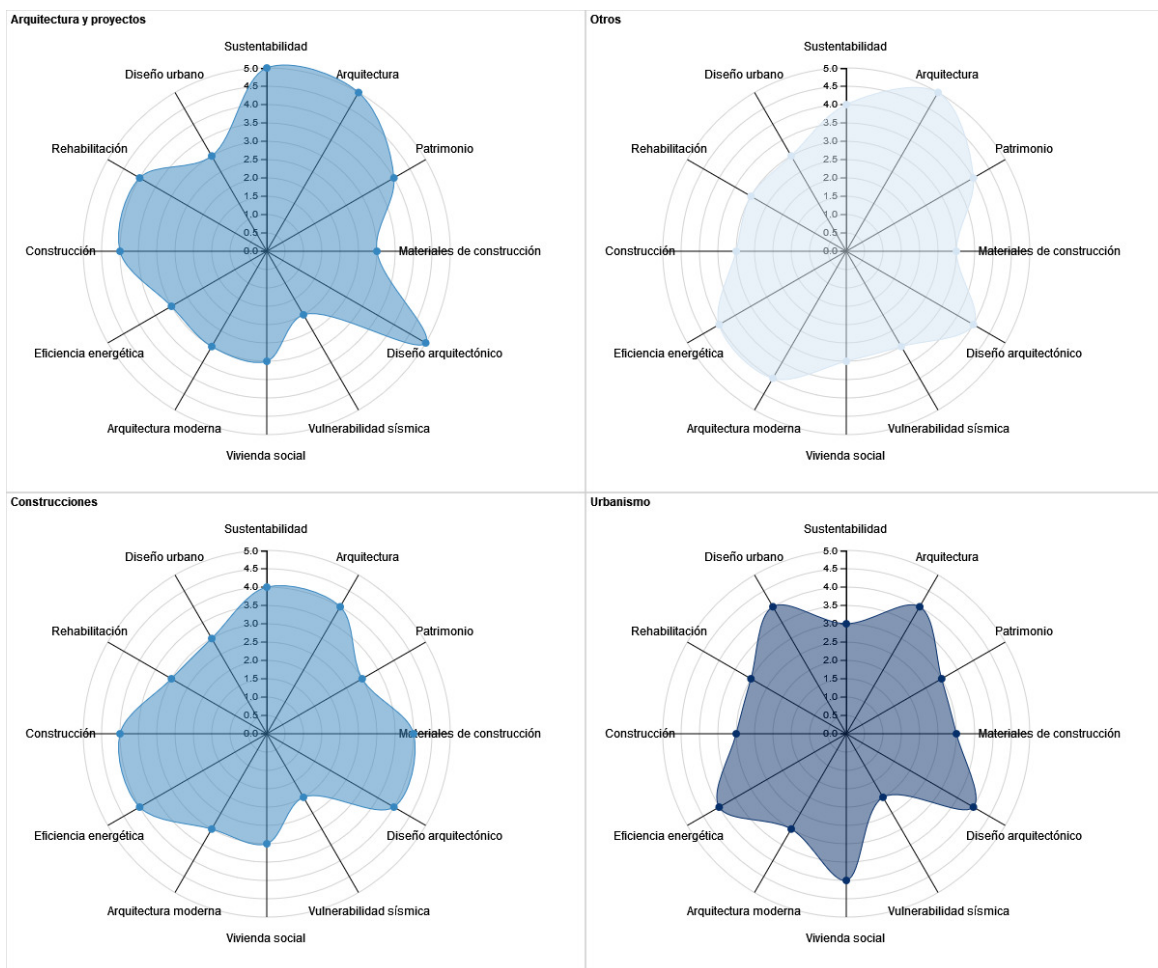

Figura 11. Relación entre grupos de perfiles docentes e intereses de investigación. Fuente: Elaboración de los autores.

utilizada para observar la evolución de la ampliación de las tecnologías digitales referidas al BIM. Con todo, es fundamental que se estudien otros tópicos para iniciar investigaciones en temas emergentes que aporten aspectos innovadores para la academia.

El análisis bibliométrico requiere de un proceso ordenado de revisión, clasificación de índicesy datos enmarcados en campos concretos del conocimiento. En el caso de "arquitectura", nos encontramos que la palabra es ampliamente utilizada en otros campos diferentes a los de la profesión, por lo que se optó por utilizar el código ASJC (2216) de Scopus que permitió limitar los procesos de búsqueda a temas directamente relacionados al campo de la Arquitectura como disciplina. Así, fueron seleccionados 1465 documentos: artículos, capítulos de libro, artículos de conferencia y revisiones de literatura.

Según los criterios establecidos para la búsqueda bibliométrica en cuanto a lo relativo a los índices $\mathrm{H}$ y el de relevancia, se constató que, en Iberoamérica, Chile y Portugal son los países de mayor producción científica en Arquitectura y que Portugal, el que mayor producción científica presenta 
tanto en publicaciones individuales como en colaboración con múltiples países. Es relevante destacar, además, que en Chile diversas universidades poseen sus propias revistas con diferentes indexaciones en bases de datos bibliográficas, lo que mejora los índices de producción científica y el desarrollo de redes de colaboración.

La curva de frecuencia de distribución de producción científica extraída desde Bibliometrix demuestra que el índice de producción en los últimos 5 años, para el caso de arquitectura, está bajo la curva, lo cual demuestra que es necesario cambiar los enfoques de investigación; una opción sería el trabajo en redes y grupos de investigación.

El mapeo científico ha conseguido identificar la dinámica de la investigación en el dominio de arquitectura en los últimos 5 años. A partir del análisis se puede determinar que las investigaciones están vinculadas a diseño, construcción y sustentabilidad, lo cual implica la necesidad de diferentes especializaciones que permitan abordar integralmente los estudios.

Finalmente, se evidencia que las universidades de diferentes países iberoamericanos dirigen sus estudios hacia tópicos asociados al concepto de sustentabilidad, lo que demuestra que este puede ser un hilo conductor de diversas investigaciones que motive la conformación y el trabajo de los grupos y redes de investigación.

\section{AGRADECIMIENTOS}

Se agradece a la Universidad Internacional del Ecuador.

\section{REFERENCIAS BIBLIOGRÁFICAS}

Agnete, V., Aina, A., Svein, K. e Ingvild, R. (2016). The establishment of formal research groups inhigher education institutions. Nordic Journal of Studiesin Educational Policy, 2016(2-3). DOI: 10.3402/ nstep.v2.33896

Aguaded, I. (2020). Publicado el nuevo SCOPUS-2019: 'Comunicar', indiscutible líder entre las revistas hispanas. Comunicar. Revista Científica de Educación y Comunicación. DOI: https://doi.org/10.3916/escuela-de-autores-136

Aria, M. y Cuccurullo, C. (2017). bibliometrix: An R-tool for comprehensive science mapping analysis. Journal of Informetrics, 11(4), 959-975. DOI: https://doi.org/10.1016/j.joi.2017.08.007

Bermeo-Giraldo, M. C., Acevedo Correa, Y., Palacios Moya, L., Benjumea Arias, M. y Arango-Botero, D. (2020). Evolución y tendencias investigativas sobre estrategias de gestión de conocimiento en instituciones de educación superior. Revista Virtual Universidad Católica del Norte, 5821(60), 202-227. DOI: https://doi.org/10.35575/rvucn.n60a11

Blakeman, K. (2018). Bibliometrics in a digital age: help or hindrance. Science progress, 101(3), 293-310. DOI: https://doi. org/10.3184/003685018X15337564592469
Chuquin J. y Salazar W. (2019). Manual para la aplicación de indicadores de produccion científica en Ecuador asociado a Scopus. Recuperado de https://bookdown.org/mabecita3/_ book2/\#elaborado-por

Ciencia Unisallle (2020). Guía de visualización de datos: Bibliometrix. Recuperado de https://ciencia.lasalle.edu.co/ recursos_bibliograficos/30

D'Amanzo, M., Mercado, M. V. y Ganem-Karlen, C. (2020). 10 preguntas de los edificios energía cero: revisión del estado del arte. Hábitat Sustentable, 10(2), 24 - 41. DOI: https://doi.org/10. 22320/07190700.2020.10.02.02

Duque, P. y Cervantes, L.S. (2019). Responsabilidad Social Universitaria: una revisión sistemática y análisis bibliométrico. Estudios Gerenciales, 35(153), 451-464. DOI: https://doi. org/10.18046/j.estger.2019.153.3389

Gallardo, E. (2016). Seminario: "Bibliometría y Revisiones sistemáticas: Una aproximación al análisis de la literatura científica en el área de Empresa. DOI: https://doi.org/10.13140/ RG.2.1.3461.7204

Grant, M. J. y Booth, A. (2009). A typology of reviews: An analysis of 14 review types and associated methodologies. Health Information and Libraries Journal, 26(2), 91-108. DOI: https://doi. org/10.1111/j.1471-1842.2009.00848.x

González C., A. y Trebilcock K., M. (2012). La sostenibilidad en el currículo de las carreras de arquitectura: Implicaciones de concepto de sostenibilidad en el perfil profesional y el plan de estudios de las carreras de arquitectura en América Latina. Hábitat Sustentable, 2(1), 26-35. Recuperado de http://revistas. ubiobio.cl/index.php/RHS/article/view/410

Manterola, C., Astudillo, P., Arias, E. y Claros, N. (2013). Revisiones sistemáticas de la literatura. Qué se debe saber acerca de ellas. Cirugía Española, 91(3), 149-155. DOI: https://doi.org/10.1016/j. ciresp.2011.07.009

Ramos-Sanz, A. (2019) Transformación de la construcción y la arquitectura en los últimos veinte años: Prospectivas y perspectivas. Análisis bibliométrico de los tópicos más desarrollados en revistas internacionales de alto impacto. Arquitecturas del Sur, 37(55), 106-125. DOI: http://dx.doi.org/10.22320/07196466.2019.37.05 5.07 .

Smith, T., Vacca, R., Krenz, H. y Mc Carty, C. (2021). Great minds think alike, or do they often differ? Research topic overlap and the formation of scientific teams. Journal of Informetrics, 15(1). DOI: https://doi.org/10.1016/j.joi.2020.101104

Snyder, H. (2019). Literature review as a research methodology: An overview and guidelines. Journal of Business Research, 104, 333-339. DOI: https://doi.org/10.1016/j.jbusres.2019.07.039

Van Raan, A. (2014). Measurement of Central Aspects of Scientific Research: Performance, Interdisciplinarity, Structure. Measurement, 3(1), 1-19. Recuperado de: http://www.cwts.nl/ TvR/documents/AvR-Measurem.pdf.

Wen, Q., Ren, Z., Lu, H. y Wu, J. (2021). The progress and trend of BIM research: A bibliometrics-based visualization analysis. Automation in Construction, 124. DOI: 10.1016/j. autcon.2021.103558 